\section{Moyamoya syndrome in an adult with essential thrombocythemia}

\author{
Marc A. Lazzaro, ${ }^{1}$ Elizabeth J. Cochran,2 \\ Demetrius K. Lopes, 3 \\ Shyam Prabhakaran 4 \\ 1Department of Neurology, Medical \\ College of Wisconsin and Froedtert \\ Hospital, Milwaukee, WI \\ 2Department of Pathology, Medical \\ College of Wisconsin and Froedtert \\ Hospital, Milwaukee, WI \\ 3Department of Neurosurgery, Rush \\ University Medical Center, Chicago, IL, \\ 4Department of Neurological Sciences, \\ Section of Cerebrovascular Disease, Rush \\ University Medical Center, Chicago, IL, \\ USA
}

\begin{abstract}
Moyamoya syndrome is a rare cerebrovascular disorder characterized by progressive occlusion of the supraclinoid internal carotid artery and proximal portions of the anterior and middle cerebral arteries resulting in an extensive network of collateralized blood vessels and producing a characteristic angiographic appearance. Although the pathophysiology is unclear, hematologic disorders have been associated with development of the moyamoya syndrome. A case report is presented. A 29 year-old female with a history of essential thrombocythemia developed progressive ischemic strokes. Angiography revealed characteristic moyamoya changes and pathologic examination showed intimal hyperplasia with scant collagen fibers and myxoid change. This is the first reported case of moyamoya syndrome in an adult patient with essential thrombocythemia demonstrating histological findings that suggest a shared pathophysiology with moyamoya syndrome in sickle cell anemia.
\end{abstract}

\section{Introduction}

Moyamoya syndrome,characterized by progressive stenosis resulting in occlusion of the intracranial circulation and extensive collateralization, produces a characteristic angiographic puff of smoke appearance. 1 The supraclinoid internal carotid artery is primarily affected and the disease may manifest with ischemic stroke, transient ischemic attack, or intracerebral hemorrhage. ${ }^{2}$
Essential thrombocythemia (ET) is a myeloproliferative syndrome defined by overproduction of platelets with predisposition to vascular occlusive events as a result of intravascular stasis and/or qualitative platelet dysfunction. ${ }^{3}$ ET has been associated with large and small vessel ischemic stroke, ${ }^{4}$ as well as micro-vessel vascular occlusive events. 5

We report a patient with essential thrombocythemia who developed multiple ischemic infarcts due to moyamoya syndrome. To our knowledge, the association between ET and moyamoya syndrome in an adult has not been previously reported.

\section{Case Report}

A 29 year-old woman presented with several brief attacks of dysarthria, aphasia, staring, and loss of consciousness. Essential thrombocythemia was diagnosed two years earlier and she was poorly compliant with hydroxyurea. Chronic inflammatory demyelinating polyneuropathy (CIDP) diagnosed one year prior resulted in bilateral foot drop. She had dietcontrolled diabetes, hypertension, migraine headaches, and childhood asthma. She did not use tobacco or illicit drugs and rarely drank alcohol. She presented bradyphrenic and abulic, followed simple commands only, but was alert. There was distal lower extremity weakness with decreased sensation and reflexes attributed to CIDP. Serum studies showed thrombocytosis $(1,080 \mathrm{~K} / \mathrm{uL})$. Magnetic resonance imaging (MRI) and angiography (MRA) revealed bilateral middle cerebral artery narrowing and acute infarcts (Figure 1A). Cerebrospinal fluid protein was elevated (114 mg/dL), consistent with CIDP, but was otherwise unremarkable including angiotensin converting enzyme and negative viral studies (herpes simplex virus and varicella zoster virus polymerase chain reaction tests). Aspirin (325 mg daily) was initiated on admission, and due to recurrent strokes clopidogrel (75 mg daily) was added and a three-day trial of methylprednisolone (1 gram intravenously daily) was implemented on day six for possible vasculitis. Angiography revealed severe stenosis at bilateral distal internal carotid arteries (ICA), proximal middle cerebral arteries (MCA), and anterior cerebral arteries (ACA) (Figure 1C), meeting Suzuki criteria for stage 1 moyamoya changes. ${ }^{1}$

During hospitalization she developed multiple new ischemic infarcts which were thought to be due to flow failure. Repeat angiography was performed twice and cyclophosphamide therapy was initiated due to persistent thrombocytosis $(500-600 \mathrm{~K} / \mathrm{uL})$. Given the multiple ischemic infarcts due to hemodynamic failure and despite multiple endovascular attempts to
Correspondence: Marc A. Lazzaro, Department of Neurology, Medical College of Wisconsin and Froedtert Hospital, 9200 West Wisconsin Avenue, Milwaukee, WI 53226, USA.

E-mail: mlazzaro2@gmail.com

Key words: stroke, moyamoya, essential thrombocythemia.

Contributions: MAL,SP, manuscript preparation and review, patient case review, imaging review; EJC, histological results interpretation, manuscript preparation and review; DKL, angiography interpretation, manuscript preparation and review. All authors contributed final approval.

Conflict of interest: the authors report no conflicts of interest.

Received for publication: 23 October 2010.

Revision received: 10 January 2011.

Accepted for publication: 16 April 2011.

This work is licensed under a Creative Commons Attribution 3.0 License (by-nc 3.0).

CC Copyright M.A. Lazzaro et al., 2011

Licensee PAGEPress, Italy

Neurology International 2011; 3:e3

doi:10.4081/ni.2011.e3

ameliorate flow, revascularization surgery with encephaloduroarteriosynangiosis (EDAS) was performed on day nineteen. She remained poorly responsive and MRI on hospital day twenty-seven revealed marked progression of bilateral MCA infarcts (Figure 1B). She died on hospital day forty-eight due to pulseless electrical activity cardiac arrest.

Serum studies for human immunodeficiency virus, erythrocyte sedimentation rate, hemoglobin electrophoresis, antinuclear antibody, rheumatoid factor, complement assay, antinuclear cytoplasmic antibody, and anticardiolipin antibodies were unrevealing. Platelet count peaked at $1099 \mathrm{~K} / \mathrm{uL}$ during the hospital course. Bone marrow biopsy was negative for Jak-2 mutation and showed an increase in megakaryocytes with multiple distinct nuclei and abnormal nuclear shapes consistent with essential thrombocytosis. No autopsy was performed. However, histological evaluation of the superficial temporal artery obtained at the time of EDAS showed intimal thickening with a narrowed lumen (Figure 2). The intima showed prominent myxoid change with intermixed smooth muscle cells and scant collagen fibers, but there was no inflammation to suggest vasculitis. The segment of middle meningeal artery, dura, and brain tissue obtained at the time of EDAS (not shown) did not show pathologic abnormalities. There was no family history of moyamoya syndrome, and the patient had no stigmata of neurofibromatosis or history of radiotherapy. 


\section{Discussion}

We report the first case of moyamoya syndrome in an adult patient with essential thrombocythemia. The only other case report of moyamoya syndrome in association with ET is that of Kornblihtt et al. in an adolescent with
ET. ${ }^{6}$ However, no pathologic tissue was reported from that patient.

The initial description of moyamoya syndrome characterized six stages of progression: narrowing of the carotid fork, initiation of the moyamoya, intensification of the moyamoya, minimization of the moyamoya, reduction of the moyamoya, and disappearance of the moy-

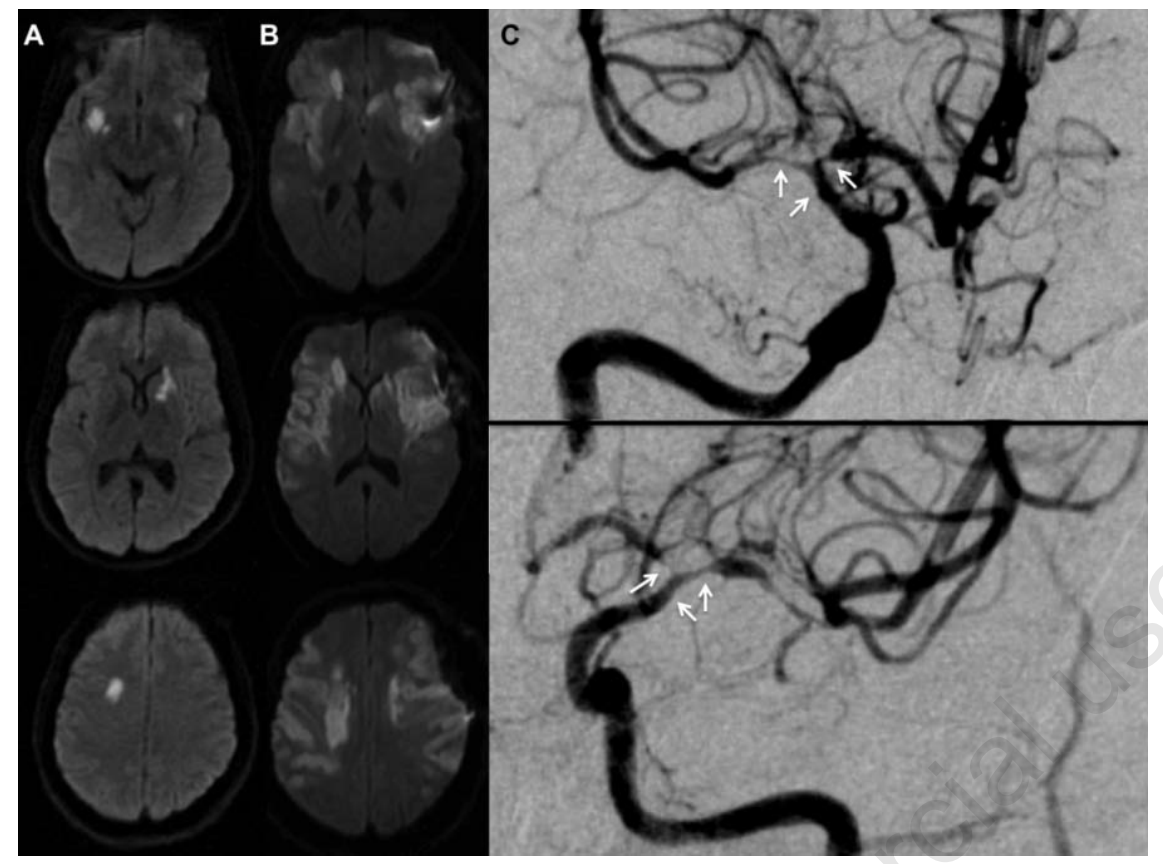

Figure 1. Diffusion-weighted imaging on admission demonstrates acute infarcts in the bilateral middle cerebral artery distributions (A), diffusion-weighted imaging on hospital day 27 shows new infarcts in the bilateral middle cerebral artery distributions (B). Cerebral angiography shows severe stenosis (arrows) of the bilateral distal internal carotid arteries, proximal middle cerebral arteries, and anterior cerebral arteries in both the right (top) and left (bottom) carotid artery injections (C).

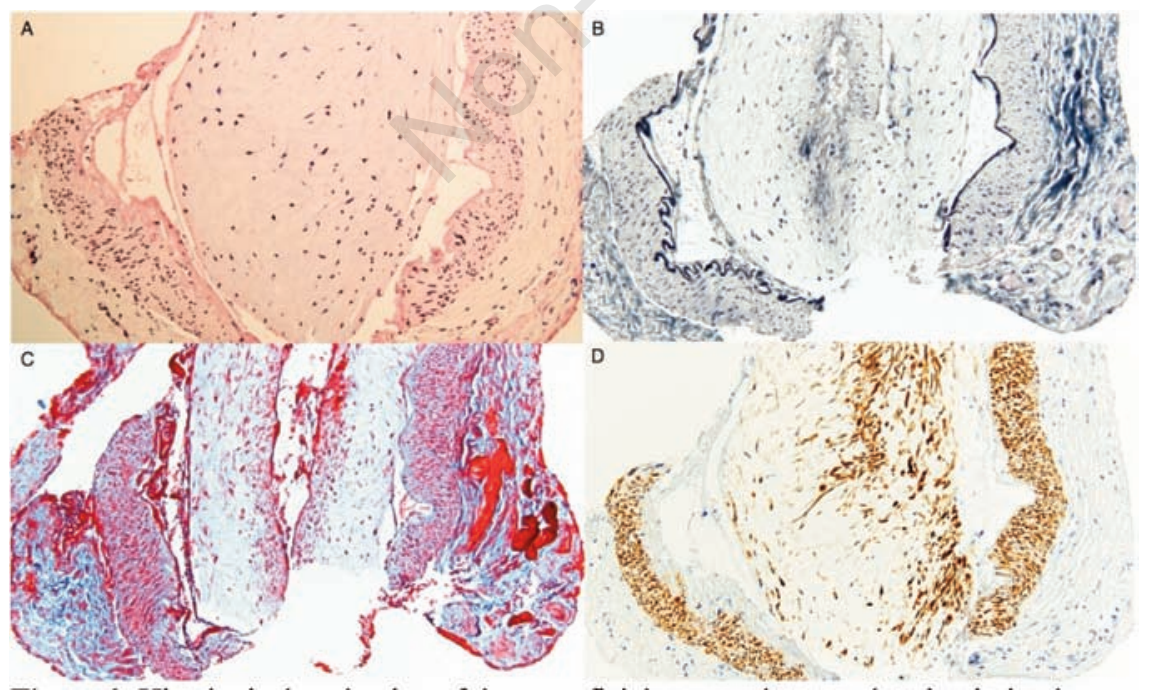

Figure 2. Histological evaluation of the superficial temporal artery showing intimal hyperplasia due to smooth muscle proliferation, scant collagen fibers, and myxoid change resulting in a narrowed lumen (H\&E (A); Elastic stain (B); Trichrome stain (C); Smooth muscle actin stain (D)). Original magnification 100x. amoya. ${ }^{1}$ Vessel occlusion in moyamoya syndrome likely results from a combination of hyperplasia of smooth-muscle cells and luminal thrombosis; however, the underlying mechanism of this process is unknown. Moyamoya syndrome has been associated with a variety of conditions and a multifactorial etiology has been suggested, including genetic causes and environmental factors such as mutations in matrix metalloproteinases and radiation. ${ }^{2}$ Yamashita et al. proposed that the development of intimal thickening may be similar to the pathogenesis of atherosclerosis and involve endothelial injury with incorporation of fibrin and platelet-rich microemboli into the arterial wall. ${ }^{7}$ The media is often attenuated with an irregular elastic lamina. ${ }^{2}$ Additionally, increased platelet-derived basic fibroblast growth factor ( $\beta$ FGF) has been reported in the cerebrospinal fluid of patients with moyamoya syndrome and may be responsible for smooth muscle proliferation. ${ }^{8}$ Moyamoya syndrome has been reported with several underlying conditions including various hematologic disorders. ${ }^{2}$

Moyamoya changes have been welldescribed in patients with sickle cell anemia (SCA) and have been reported in up to $43 \%$ of pediatric patients with SCA who have strokes. ${ }^{9}$ An early description of moyamoya syndrome in SCA patients suggested that occlusion of the vasa vasorum by sickled cells leads to ischemia, progressive intimal and medial-wall proliferation, and vessel occlusion. ${ }^{10}$ Largeartery intimal hyperplasia in SCA has also been thought to develop from endothelial irritation, intimal edema, and proliferation at arterial bifurcations as a response to repeated sickle cell crises. ${ }^{11}$ Intimal hyperplasia has been reported in SCA patients in both intraand extracranial vascular beds. 12

In thrombocythemia, a hyperviscosity state secondary to thrombocytosis, platelet function abnormalities, abnormal platelet morphology, abnormal arachidonic acid metabolism, and increased neutrophil-platelet aggregation, are all implicated in the thrombotic state. 13 These changes, along with elevated levels of bFGF, may contribute to a pro-thrombotic milieu in ET that predisposes to endothelial injury and leads to intimal hyperplasia resulting in progressive occlusion and thrombosis of mediumsized arteries. As systemic conditions that can affect all vascular beds, ET and other hematologic disorders would be expected to cause generalized intimal hyperplasia and thrombosis which would not be restricted to intracranial vessels. Our pathologic finding of extracranial intimal hyperplasia supports this hypothesis and provides the first evidence of a shared pathophysiology between ET and SCArelated moyamoya syndrome. 


\section{References}

1. Suzuki J, Takaku A. Cerebrovascular "moyamoya" disease. Disease showing abnormal net-like vessels in base of brain. Arch Neurol 1969;20:288-99.

2. Scott RM, Smith ER. Moyamoya disease and moyamoya syndrome. N Engl J Med 2009;360:1226-37.

3. Hart RG, Kanter MC. Hematologic disorders and ischemic stroke. A selective review. Stroke 1990;21:1111-21.

4. Arboix A, Besses C. Cerebrovascular disease as the initial clinical presentation of haematological disorders. Eur Neurol 1997;37:207-11.

5. Michiels JJ, Berneman Z, Van Bockstaele D, et al. Clinical and laboratory features, pathobiology of platelet-mediated throm- bosis and bleeding complications, and the molecular etiology of essential thrombocythemia and polycythemia vera: therapeutic implications. Semin Thromb Hemost 2006;32:174-207.

6. Kornblihtt LI, Cocorullo S, Miranda C, et al. Moyamoya syndrome in an adolescent with essential thrombocythemia: successful intracranial carotid stent placement. Stroke 2005;36:E71-3.

7. Yamashita M, Oka K, Tanaka K. Cervicocephalic arterial thrombi and thromboemboli in moyamoya disease--possible correlation with progressive intimal thickening in the intracranial major arteries. Stroke 1984;15:264-70.

8. Yoshimoto T, Houkin K, Takahashi A, Abe H. Evaluation of cytokines in cerebrospinal fluid from patients with moyamoya disease. Clin Neurol Neurosurg 1997;99: S218-20.
9. Dobson SR, Holden KR, Nietert PJ, et al. Moyamoya syndrome in childhood sickle cell disease: a predictive factor for recurrent cerebrovascular events. Blood 2002; 99:3144-50.

10. Stockman JA, Nigro MA, Mishkin MM, Oski FA. Occlusion of large cerebral vessels in sickle-cell anemia. N Engl $\mathrm{J}$ Med 1972;287:846-9.

11. Merkel KH, Ginsberg PL, Parker JC Jr, Post MJ. Cerebrovascular disease in sickle cell anemia: a clinical, pathological and radiological correlation. Stroke 1978;9:45-52.

12. Francis RB Jr, Johnson CS. Vascular occlusion in sickle cell disease: current concepts and unanswered questions. Blood 1991;77:1405-14.

13. Schafer AI. Bleeding and thrombosis in the myeloproliferative disorders. Blood 1984; 64:1-12. 